\title{
Summary
}

The author offers a new method of operation for correcting severe cases of spastic entropion following total tarsectomy. The method consists in a cartilage of corresponding size being taken out of the concha auriculae and laid in the bed prepared in the lid, to replace the tarsus removed by total tarsectomy. The operation consists of three steps described in the text and illustrated in the attached Table.

The author has operated in the above manner on five cases with consistently good results. The patients have been demonstrated before the Haifa Society of Oculists and the Jewish Medical Society in Haifa.

\section{ANTEPOSITIO CONJUNCTIVAE FORNICIS. OPERATION IN SEVERE CASES OF SPRING CATARRH*}

\author{
BY \\ N. I. SHIMKIN \\ HAIFA, PALESTINE
}

IN Palestine, as well as in Egypt, spring catarrh occurs the whole year round, i.e., both during the cold and hot time of the year. But in the hot season, the clinical diagnosis of spring catarrh is not always easy, owing to the presence of trachoma and acute seasonal conjunctivitis in different forms. An immediate clinical determination of the diagnosis becomes especially difficult with the papillary form of trachoma. The exact setting up of a differential diagnosis among the trachoma highly spread in Palestine and the comparatively rare cases of spring catarrh, is possible only after curing acute conjunctivitis, the aetiology and epidemiology of which in Palestine have been expounded by me in a whole series of other articles (Shimkin, Harefua (Hebrew), 1923. Ann. d'Ocul., 1924. Brit. Jl. of Ophthal., 1926. Compte Rendu de Congrès International à Caire, 1931. Tome III).

While according to the data of various authors, up to 65 per cent. of the Arabic population in Palestine is affected with trachoma, and in the South of Palestine, in the villages, even up to 95 per cent., there are no more or less comprehensive statistics of the frequency of spring catarrh in Palestine.

\footnotetext{
* Paper read on December 9,1937. at the Fifteenth Concilium Ophthalmologicum in Cairo, Egypt
} 
The statistics of the number of out-patients in my clinic do not throw much light on this question, as the Arabic population applies to an ophthalmologist for special aid only in most difficult cases of spring catarrh, when itching, epiphora, photophobia, and sometimes blepharospasm deprive the patient of the possibility of working.

On examining such patients, one can see that papillae, hard as glass, densely cover all the conjunctiva of the tarsi up to the extreme border of the lids. Sometimes these papillae have grown so violently that they turn out up to the intermarginal line, forming such a thick layer of conjunctiva that it is 2-3 times thicker than the tarsus lying under it.

This abnormal thickening of conjunctiva at the lid border is common in spring catarrh, and may serve as one of the pathognomonic symptoms for differential diagnosis between spring catarrh and trachoma, but only after an acute conjunctivitis has been cured. Up till now little attention has been paid to this symptom. The normal lid border consists of three layers :-(1) skin, (2) intermarginal space, and (3) a tarsus with the thinnest of conjunctiva not seen by the naked eye.

In spring catarrh this free edge of the lid border consists of four layers :- (1) skin, (2) intermarginal space, (3) tarsus, and (4) a thick conjunctiva, the thickness of which is 2-3 times greater than that of the lid-tarsus. The presence of such a thick conjunctiva should always suggest to the physician its true cause, namely, spring catarrh.

The Arabic population, peasants and workers, consult an oculist expecting quick medical aid and relief; but spring catarrh is among those diseases of the conjunctiva in which medicamental therapy may bring forth a temporary relief but not healing. Hopes laid upon radiotherapy have only partly come true, for curing slight and medium stages of spring catarrh, but in severe forms of spring catarrh radiotherapy has proved ineffectual (Stallard, ${ }^{1}$ Wilson, ${ }^{2}$ El Tobgy ${ }^{3}$ ).

The operative treatment in severe cases of spring catarrh recommended by all manuals, consists either in excising the papillae on the conjunctiva of the tarsus or in their thermo- or galvanoor diathermo-cauterisation. As a palliative means, this operative interference alleviates the most unpleasant symptoms of the patient for a very short time; but the papillae on the conjunctiva of the tarsus soon grow again, and the patient returns to the doctor with the same complaints as before.

Axenfeld suggests partial tarsectomy, in which operation both

(1) Radiant Energy, Brit. Jl. Ophthal. (2) Annual Report, Giza Memorial Ophthalmic Laboratory, 1930 and 1933. (3) Folia Ophthal. Orientalia, 1933. 
a part of the affected conjunctiva and the tarsus lying under it are removed.

But in severe forms of spring catarrh, all the conjunctiva of the tarsi up to the very lid border is affected; therefore, a partial tarsectomy brings little benefit in those cases, as the papillae remaining on the tarsal conjunctiva bring forth the same hard manifestations of itching, burning, lacrimation and photophobia. The combined Kuhnt-Heisrath tarsus excision, in which all the tarsus with the conjunctiva is removed $2 \mathrm{mms}$. down from the lid border, gives good results and very quickly frees the patient from sore sensations caused by this condition. But sometimes the papillae on the narrow part of the tarsus left, even when removed with a knife, during the operation, grow again and cause the same painful sensations, considerably lighter, however, than before.

But the application of combined Kuhnt-Heisrath excision for the therapy of spring catarrh is not justified either by the clinical or pathological picture of the disease.

On clinical examination of the excised portion of the lid tarsus, with the adjoining conjunctiva, the normal unthickened tarsus and the highly thickened tarsal conjunctiva attract the surgeon's attention.

The pathological researches of many authors have proved that in spring catarrh the tarsus with the Meibomian glands and their excretory ducts are fairly normal. As to the substance of the pathological process in the conjunctiva tarsi, it is seen that "the papillae of the conjunctiva tarsi originate owing to the growth and later hyaline degeneration of the subconjunctival tissue. Especially under the epithelium there is such a hyaline layer present, which gives the bluish-white appearance" (E. Fuchs, Handbuch f. Augenheilk.).

But if the lid tarsus in spring catarrh is normal, why should it be excised? If the locus morbi is only in the tarsal conjunctiva and subconjunctival tissue, then it is natural to remove only the affected tissue, and to cover the defect produced with a healthy conjunctiva. If there is enough conjunctiva of the fornix for covering the defect of the tissue in the combined Kuhnt-Heisrath tarsus excision, then the same conjunctiva will be also sufficient for covering the denuded area of the tarsus, after the removal of the diseased hyaline degenerated conjunctiva from its surface.

These considerations have involuntarily been called forth after each performance of the combined Kuhnt-Heisrath tarsus excision for surgical interference in spring catarrh by me. When spring catarrh was being treated by operation, it seemed quite logical to me to leave the normal tarsus in its place, to remove all the affected conjunctiva from its surface, and to cover the denuded 
area with the healthy conjunctiva of the fornix forward up to the border of the lid. (Antepositio Conjunctivae Fornicis.)

If the denuded tarsus after the removal of the diseased conjunctival tissue is not covered with healthy conjunctiva, then after the regeneration of the epithelium on the surface of the tarsus, the papillae, characteristic of spring catarrh, grow up again out of the islets of the conjunctival tissue which have remained; and all morbid symptoms of this disease return.

The name which I give to this operation, i.e., Antepositio Conjunctivae Fornicis, corresponds to the actual performance of the operation. The deeply separated-off healthy conjunctiva fornicis is drawn forward on to the healthy tarsus of the lid, after removing the affected tarsal conjunctiva with diseased subconjunctival tissue.

Instruments required.-Jaeger's horn spatula, needle-holder, two silk sutures with one needle for drawing up the lid, and three silk sutures with needles at each end for three loop sutures. Forceps, a sharp scalpel, and half-bent scissors.

Anaesthetic.-As for the combined Kuhnt-Heisrath tarsus excision : 1.5 c.c. 2 per cent. novocaine + adrenalin are injected under the conjunctiva of the fornix, and 0.5 c.c. of the same solution under the skin of the lid border.

\section{The Operation consists of Five Steps:}

\section{First Step. Inserting two Sutures Drawing the Everted Upper} Lid on Spatula (Fig. 1a).

The needles are inserted in the skin of the lid border and brought out along the intermarginal line (Fig. 1b). One suture is placed on the margin of the middle and the outer thirds of the lid border; and the other suture, on the margin of the middle and the inner thirds. These two sutures are very convenient for strong retraction of the lid turned upwards on Jaeger's spatula, they do not touch the conjunctiva at all, and therefore, all the lid border conjunctiva, affected with papillary growth, is clearly seen. These sutures do not hinder the removal of the conjunctiva at its very margin which is impossible on applying any forceps to the lid border.

Second Step. Incision of the conjunctiva of the fornix along its whole length.

The incision is made $2 \mathrm{mms}$. below the demarcation line, dividing the affected tarsal conjunctiva, from the healthy conjunctiva of the fornix (Fig. 1c). The line of demarcation of the healthy conjunctiva of the fornix, made anaemic with adrenalin, stands out 


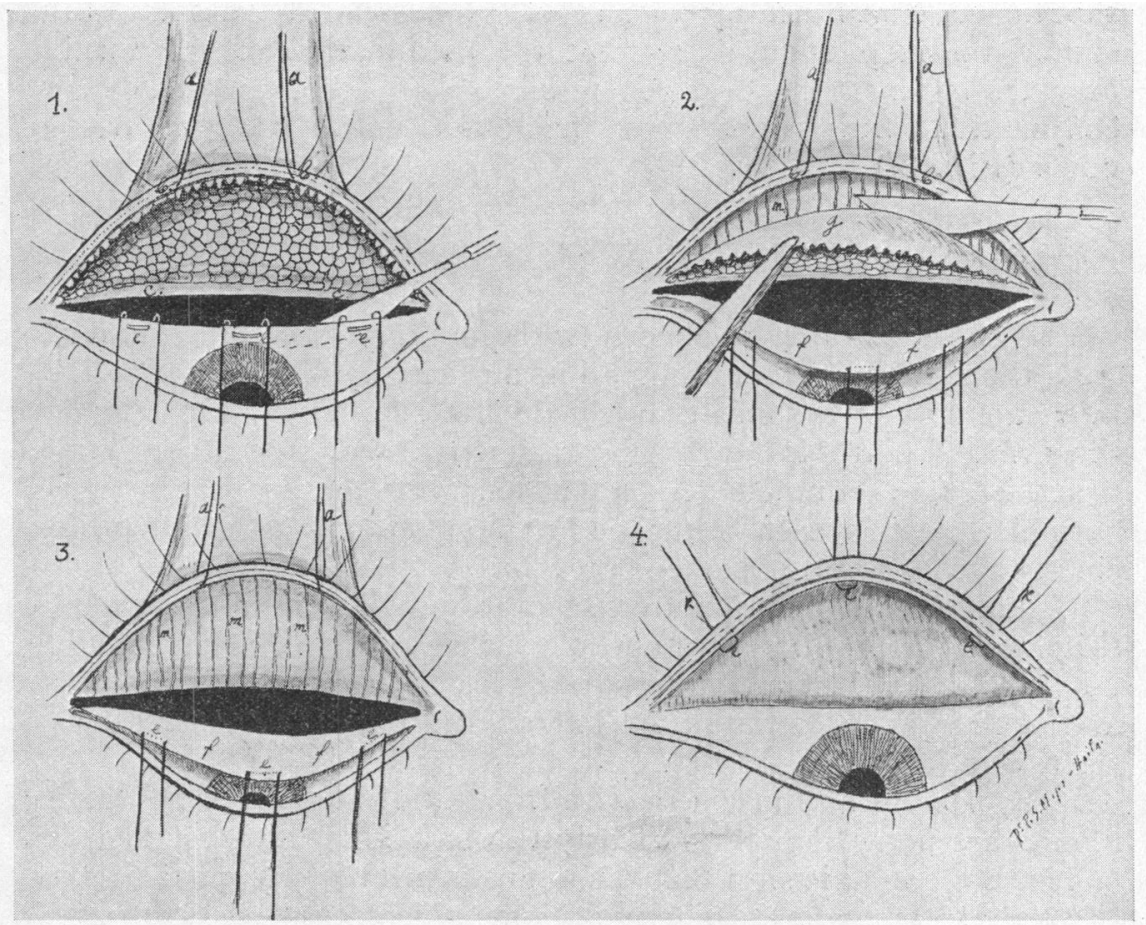

clearly, if the lid is drawn tightly on Jaeger's spatula. Immediately after the incision, the bulbar part of the conjunctiva contracts and goes down. The subconjunctival tissue, denuded after the incision, is shown in figures as a wide black strip (Fig. 1d). Immediately after the incision in the conjunctiva, still not separated from the underlying tissues, three loop sutures are applied on its bulbar part, in its superficial layers, $1 \mathrm{~mm}$. away from its border (Fig. 1e); one loop suture in the middle, one on the margin of the middle and outer thirds, and one on the margin of the middle and inner thirds. Thanks to the conjunctiva still adhering to the underlying tissue, the loop sutures applied at the very border of the bulbar conjunctiva, in the middle and at the sides, will precisely correspond to the middle and to the outer and inner thirds of the lid border.

Third Step. Drawing the conjunctiva of the fornix down and outwards, Separate it off carefully from the underlying tissue by means of blunt-headed scissors (Fig. 2f, Fig. 3f).

The conjunctiva thus separated off should be thin and not contain any underlying tissue. This separation of the conjunctiva 
should be continued by means of blunt-headed scissors; and should be deep enough, so that the conjunctiva of the fornix may freely cover the denuded tarsus. The deeply separated off conjunctiva fornicis is everted, inside out, and is hanging freely down (Fig. 2f).

Fourth Step. The removal of the tarsal conjunctiva (Fig. $2 g$, Fig. $3 m$ ).

The tarsal conjunctiva affected with papillary growth is removed from the extreme border along all its surface, by means of saw-like movements with a sharp scalpel laid flat side down. In Fig $2 g$ the everted part of the tarsal conjunctiva with the underlying tissue is seen. Immediately after removing the conjunctiva the normal tarsus is seen with the Meibomian glands glimmering clearly through its substance (Fig. $3 \mathrm{~m}$ ).

Usually, all the conjunctiva separates easily from the tarsus, from top to bottom, along its whole surface. The surgeon should take exceptional care that no traces of the affected conjunctiva and subconjunctival tissue are left on the border and surface of the tarsus.

If, by separating the conjunctiva from the tarsus, the Meibomian glands are not glimmering clearly through its substance, then it is a pathognomonic sign that the tarsus is not healthy, and mostly affected by trachoma in different stages not recognised before the operation. In such a case it is better to cut out the tarsus and perform a classical Kuhnt-Heisrath combined tarsus excision.

Fig. $3 m$ shows what the normal tarsus is like, when all the affected tissue of the conjunctiva is removed from it and also, how deeply the bulbar conjunctiva is separated off and turned down (Fig. 3f).

(The letters indicate : $e$-the loops of the sutures seen through; $f$-conjunctiva fornicis turned out ; $m$-Meibomian glands of the tarsus.)

Fifth Step. Carrying stitches through the tarsus and leading them out between the eyelashes (Fig. 4e, Fig. 4k).

As the correct application of sutures in the operation of Antepositio Conjunctivae Fornicis operation is very important for the cosmetic and therapeutic results of the operation, I consider it necessary to repeat the following once more : I apply the sutures on the border of the bulbar conjunctiva, not yet separated off, immediately after the incision. The needles of the three loop sutures are inserted $1 \mathrm{~mm}$. away from the border of the conjunctiva, the width of the loop of each stitch on the exterior side of the conjunctiva being $2 \mathrm{~mm}$.; the loops lie on the middle of the 
conjunctiva and on the outer and inner third (Fig. 4e). Each needle of each loop stitch is pierced into the thickness of the tarsus $1 \mathrm{~mm}$. away from its border and is carried slantwise, so as to go out between the eyelashes. In Fig. $4 a$ is clearly seen : (1) the position of the loops on the conjunctiva (4e), (2) their exit between the eyelashes $(4 k)$. Due to the above method of applying the sutures, each needle passes slantwise in the thickness of the tarsus and has a compact tissue along all its course, thanks to which tight tying of the knots is not required, as the threads are held by the dense tissue of the tarsus. Resulting from the passage of the threads aslant from below upwards, the conjunctiva, being drawn by them, is drawn up to the extreme border of the lid, and finally, the above-mentioned leading of the sutures, after they are tied, gives a slight ectropion of the border of the upper lid. These sutures applied by me in the Kuhnt-Heisrath operation always give slight lid ectropion. They were demonstrated by me at the XIIIth Conference of Russian Ophthalmologists in Moscow in 1913, and elaborated on corpses at the Institute of Operative Surgery of Novorosseesk University (Odessa) directed by Professor N. K. Lissenkov and published in Westnic. Ophthal., No. 6, 1914.

After inserting all the six needles, we receive three loop sutures. Drawing these sutures up, we draw the healthy fornix conjunctiva forwards on the denuded tarsus. Fig. 4 clearly demonstrates the result of the operation and position of the conjunctiva drawn on.

After the operation, a binocular bandage is applied for two days. On the third day the sutures are taken out, and again a bandage is applied for another two days. After four days the patient is sent home. The unpleasant manifestations of spring catarrh disappear entirely and immediately. The operation antepositio conjunctivae fornicis described has been applied by me already for eight years, in cases of severe forms of the disease.

Twenty-nine patients altogether have been operated upon by me on both eyes :- -21 men and 8 women, between the ages of 20-39 years. I have observed a series of patients seven years after the operation. All the time they have not suffered from any of their former morbid sensations; there was no itching, burning or epiphora. The conjunctiva of the fornix drawn forward, covering the tarsus was free of all papillae; the lid border had a normal appearance.

In those cases where the lid border has not been thoroughly cleaned of papillary growths, and the conjunctiva has not been drawn up to the extreme lid border, separate papillae appeared again, but the itching was so slight that the patients returned to their work. 
In view of the good results obtained by me in treating severe cases of spring catarrh by means of the operation "antepositio conjunctivae fornicis," and the simplicity of its performance, I beg to draw it to the attention of my colleagues.

In conclusion, it should also be mentioned that by means of the above operation the patient gets rid of morbid sensations caused by spring catarrh in the minimum of time (5-6 days). Patients on whom I operated belonged to the peasant or working class who cannot afford to spend much time for treatment for economic reasons, and were, therefore, very pleased to have got rid so rapidly of the disease which had been torturing them for several years.

\section{Summary}

In severe forms of spring catarrh neither the medicaments nor radio-therapy gives a cure. The surgical interference in the form of excision of the hard papillae of the conjunctiva of the tarsus, or their cauterisation gives only a temporary improvement. The partial excision of the tarsi advised by Axenfeld gives very little improvement, because the remaining tarsal conjunctiva is covered with papillae of spring catarrh. The combined Kuhnt-Heisrath tarsus excision applied by some authors is not justified either by the clinical or the pathological picture of the disease.

The author points out that pathological investigations have shown that in spring catarrh the tarsus of the lid is quite healthy, and the affection consists in the disease of the conjunctiva and subconjunctival tissue only, in consequence of the abnormal growth of this tissue and its hyaline degeneration in the region of the tarsal conjunctiva.

In view of the fact that the tarsus of the lid in spring catarrh is healthy, and the tarsal conjunctiva only is diseased, the author recommends careful excision of all the conjunctiva and subconjunctival tissue from the whole surface of the tarsus, beginning at the extreme lid border, and replacing it with healthy conjunctiva of the fornix. The author calls this operation " antepositio conjunctivae fornicis," as the conjunctiva of the fornix, separated deeply down towards the eyeball, is drawn forward on the tarsus, freed from the morbid conjunctiva and subconjunctival tissue.

The author has operated on 29 cases of severe forms of spring catarrh ( 21 men and 8 women aged between 20-39 years) according to the above method, during the past seven years. The results obtained proved quite satisfactory both from the therapeutic and cosmetic point of view, and no relapses have been observed during 
the period of observation. Separate cases were observed seven years after the operation.

The operation consists of five steps described in the text and drawn in the attached Table.

The steadiness of the therapeutic results obtained, ease of the performance of the operation, the short duration of the postoperative treatment (5-6 days), all these data are, in the author's opinion, sufficient to recommend to oculists the application of the operation-antepositio conjunctivae fornicis-in severe forms of spring catarrh, not yielding to any other treatment.

\section{A CASE OF ANGIOMA RETINAE}

BY

DR. I. FEIG

haIFA, PALESTINE

Angroma retinae is an extremely rare anomaly or disease-it is in fact both of these simultaneously. Leber classifies the primary angioma and angiomatous degeneration of the retina together as Hippel-Czermak's disease of the retina, and gives a survey of 28 cases with which he was familiar. Czukrasz reports that during a period of 15 years at the Debrecen Hospital only two cases of angiogliosis retinae out of 100,000 cases of diseases of the eyes were encountered. Recently isolated cases of angiomatosis retinae, as well as several cases described as arterio-venous aneurysm, and one case of angioma of the choroid have been reported. Since, however, I have never found any case mentioned in literature available to me, similar to the angioma of the retina which was under my personal observation, I feel justified in recording the following case.

In June, 1937, Mrs. K.K., a specialist in children's diseases, came to consult me, and gave me the following history : 45 years of age, married, has no children (cause of childlessness known but of no significance as far as the disease of the eye is concerned), father died at the age of 67 years, of sepsis resulting from hypertrophy of the prostate, mother aged 74 years still in good health, five brothers and sisters, one of whom was killed in the Great War, the others in good health except for one stepbrother (descending from a different father) who has recently been suffering from diabetes. There have been no intermarriages in the family, nor have any diseases of the eyes been known of heretofore. Patient only had measles and mumps as a child. Married at age of 28 years, never gave birth, but had a doubtful abortion at one time. 